\title{
Las fuentes incas: paisaje cultural de dominio en la provincia de Cañar, Ecuador
}

Artículos originales: ARQUEOLOGÍA

Recibido: 17/02/2021

Aprobado: 12/06/2021

Publicado: $14 / 10 / 2021$
Dayuma Guayasamín Ortiz Universidad Nacional Mayor de San Marcos ${ }^{1}$ dayuma.go@hotmail.com

\begin{abstract}
RESUMEN
La actual provincia de Cañar en Ecuador fue el centro político y económico de la cultura Cañari durante el período de Integración (500 a 1500 d.C). Con la expansión del Tawantinsuyo, los Incas establecieron varias estrategias de dominio político e ideológico sobre las poblaciones locales del Chinchaysuyo, las cuales se expresaron a través de una apropiación cultural de ciertos espacios geográficos especiales, utilizando una arquitectura y toponimia específica que permitía replicar la idea de «Otro Cuzco». En los complejos arqueológicos de Ingapirca y Coyoctor en Cañar, se encuentran estructuras talladas en afloramientos rocosos conocidas como Bańos del Inca. En realidad, se trata de Fuentes de filiación Inca que son lugares de culto al agua y de purificación, pero también espacios desde donde se legitimó la dominación Inca a través del mito de origen y la implantación de festividades como la Situa.
\end{abstract}

Palabras Clave: Fuentes Incas, dominio Inca, Ingapirca, Coyoctor, mito de origen, Situa

\section{The Inca fountains. A cultural landscape of dominance in the province of Cañar, Ecuador}

\begin{abstract}
The current province of Cañar in Ecuador was the political and economic center of the Cañari culture during the Integration period (500 to $1500 \mathrm{AD}$ ). With the expansion of the Tawantinsuyo, the Incas established different strategies for political and ideological dominance over the local populations of Chinchaysuyo, which were expressed through a cultural appropriation of certain geographic spaces, using a specific architecture and toponymy that allowed replicating the idea of 'another Cuzco'. In the archaeological complexes of Ingapirca and Coyoctor in Cañar, there are carved structures in rocky outcrops known as Baños del Inca. In reality, these were Inca's structures and places for purification and worship of water. Also, spaces to legitimize Inca domination through the myth of origin and the implementation of festivities such as Situa.
\end{abstract}

KeYworDs: Inca sources, Inca domain, Ingapirca, Coyoctor, origin myth, Situa

1 Este artículo es una versión sintetizada de la tesis de maestría de Arqueología Andina de la Universidad Nacional Mayor de San Marcos titulada Apropiación de un espacio geográfico, expresada en la ubicación y diseño de los Baños del Inca de los complejos arqueológicos de Coyoctor e Inhapirca, provincia del Cañar, Ecuador. 


\section{Introducción}

J unto con la corriente posprocesualista de los años 80 , se comienza a estudiar de manera más sistemática la capacidad del Ser Humano para razonar y decidir en la transformación de su entorno natural satisfaciendo sus necesidades. Autores como Ian Hodder (1990), Michael Shanks y Christopher Tilley (1990) así como Robert Preucel (1990) destacan dentro de este movimiento con sus estudios relacionados a la distribución arqueológica de los yacimientos en determinado espacio geográfico (método estadístico), estudios fenomenológicos de los objetos arqueológicos y la evolución del paisaje. Es en este contexto que surge la Arqueología del Paisaje, la cual se interesa en la relación entre Ser Humano y Medio ambiente en cuanto a la apropiación cultural de espacios naturales, con un conjunto de conceptos teóricos y metodológicos que enfatizan sobre «entornos, poblamiento, espacios agrarios y espacios simbólicos» (Criado 1999).

En el caso de Ecuador, los estudios sobre arqueología del paisaje han sido poco tratados. Destacándose los siguientes: Persistencias y rupturas en el uso del espacio. Reflexiones sobre identidad y áreas de actividad (Ugalde et al. 2009), Rumipamba bajo la sombra del Pichincha (Constantine 2013) y Los petroglifos del Catazho, Morona Santiago: Una aproximación a su estudio desde la arqueología del Paisaje (Granja 2014). Sin embargo, no se ha realizado un análisis desde esta corriente teórica aplicado al dominio Inca expresado en sus evidencias arquitectónicas, sobre todo de ciertas estructuras talladas en afloramientos rocosos y que son un componente simbólico importante del proceso cultural. Por esta razón, en este estudio se ha decidido tratar el concepto de una "construcción simbólica del paisaje» en dos sitios arqueológicos determinados, que fueron parte primero de una ideología Cañari y posteriormente Inca. Las culturas prehispánicas en el Ecuador, durante el período de Integración (500-1500 d. C), han tenido un marcado y complejo sistema socio-político; y en particular, la cultura cañarí, ubicada en las actuales provincias de Cañar, Azuay y parte de Chimborazo, la cual tuvo un proceso histórico activo de contacto con los Incas, desde 1450, aproximadamente. En efecto, durante la conquista Inca, se llevó a cabo un proceso de dominio político, económico y social, pero sobre todo ideológico hacia los pueblos conquistados. Esta estrategia de dominio es evidenciada en la superposición de monumentos sagrados sobre los monumentos o asentamientos nativos. Sin embargo, los estudios al respecto han sido, en su mayoría, relacionados con arquitectura monumental, sin reparar en otras evidencias arqueológicas que podrían estar revelando una situación ideológica más compleja. En efecto, en la provincia del Cañar, en una zona límite entre el cantón El Tambo y el cantón Cañar, en la cuenca alta del río Cañar, específicamente, existen dos complejos arqueológicos muy importantes, distantes $5 \mathrm{~km}$ el uno del otro: Coyoctor e Ingapirca. Estos complejos, ubicados a 2970 y 3100 m.s.n.m. respectivamente, presentan evidencia de arquitectura monumental, así como estructuras más pequeñas talladas en afloramientos rocosos naturales, conocidas como Baños del Inca. El tema de estas estructuras labradas en roca ha pasado casi desapercibido en Ecuador. Si bien existen estudios sobre el contexto cultural dentro del cual se supone están inmersos, no se ha realizado estudios específicos sobre éstos. En este aspecto, pasaron a ser parte de un adendum en la explicación funcional del sitio monumental, relacionado la mayor parte con hipótesis no comprobadas pero empleadas para la atracción turística. Es en este contexto, que se enmarca el presente estudio, el cual pretende aportar con el aspecto científico del emplazamiento y diseño de estas evidencias arquitectónicas englobadas dentro de la cosmovisión andina. Es decir, se trata de aportar al entendimiento de estas estructuras desde una visión del paisaje cultural, no como un hecho fortuito, sino con una lógica cultural detrás de su ubicación y de los elementos arquitectónicos que la componen. Para este trabajo, se han realizado varias prospecciones y visitas de campo, a partir de los antecedentes y bibliografía recopilados, con la intencionalidad de establecer un aporte metodológico de los criterios a utilizar en la identificación de sitios simbólicos andinos y de dominio que permitan establecer pautas de análisis en el entendimiento de los sitios arqueológicos a través de un enfoque de Arqueología del Paisaje. Las observaciones realizadas, por lo tanto, son producto de un trabajo de campo.

Sin duda, la confluencia entre los espacios naturales y los espacios construidos por el Ser Humano en aspectos tanto físico como ideológico generan constructos que derivan en un paisaje cultural. Pero ¿De qué manera la localización y posterior diseño de 
estructuras conocidas como Baños del Inca, en los sitios arqueológicos de Coyoctor e Ingapirca, en la provincia de Cañar (Ecuador), reflejan la apropiación cultural de un espacio geográfico? El objetivo de esta investigación es entender la intencionalidad cultural que hace que un espacio natural se vuelva sagrado y esta nueva ideología se exprese a través del emplazamiento y construcción arquitectónica de los Baños del Inca.

\section{Marco teórico y metodológico}

Para abordar este trabajo de investigación se utilizó el análisis desde la Arqueología del Paisaje que estudia la relación entre el Ser Humano y el Medio Ambiente basado en el concepto de "espacio», la cual se enmarca dentro del movimiento teórico conocido como Postprocesualismo surgido en la década de los años 80 en contraposición a la Nueva Arqueología. La Arqueología Postprocesual busca entender a las sociedades a través de los conflictos entre los grupos (Alonso 2009) e intenta analizar la conciencia práctica (Schuster 1993) que mueve al Ser Humano a transformar su entorno de acuerdo a una necesidad básica. Por lo tanto, Arqueología del Paisaje, toma al espacio como punto de partida en el proceso de análisis social y simbólico de una sociedad o del contacto entre varias sociedades.

Para hablar de arqueología del paisaje es necesario entender lo que es el paisaje cultural. La definición de Sauer (1925: 45) es hasta ahora la más utilizada:

El paisaje cultural se crea, por un grupo cultural, a partir de un paisaje natural. La cultura es el agente, el área natural el medio, y el paisaje cultural el resultado. Bajo la influencia de una cultura dada, que cambia ella misma con el tiempo, el paisaje sufre un desarrollo, atraviesa fases y probablemente alcanza, por último, el fin de su ciclo de desarrollo. Con la introducción de una cultura diferente -es decir, ajena- se produce un rejuvenecimiento del paisaje, o uno nuevo que se sobrepone a los restos del antiguo.

En este intento de estudiar la relación entre el Ser Humano y el medio ambiente, Felipe Criado propone:
El estudio de este fenómeno desde el punto de vista arqueológico [el cual] constituye de hecho la arqueología de paisaje. Bajo esta denominación podemos entender un programa de investigación orientado hacia el estudio y reconstrucción de los paisajes arqueológicos o, mejor, el estudio con metodología arqueológica de los procesos y formas de culturización del espacio a lo largo de la historia. (Criado 1999: 12)

Por lo tanto, Criado plantea concebir al paisaje como «El producto sociocultural creado por la objetivación, sobre el medio y en términos espaciales, de la acción tanto de carácter material como imaginario» (Criado 1999: 5).

Dentro del análisis de los paisajes se enfatizará en el estudio de los espacios simbólicos de las sociedades andinas. Esta rica cosmovisión del pensamiento andino permite entender la importancia de algunos espacios naturales y la modificación de estos como respuesta a una ideología cultural, expresada a través de varios tipos de arquitectura, pero también mediante elementos intangibles.

Los sitios, lugares o paisajes sagrados muestran, además, temas recurrentes en perspectiva global: la concentración en cerros, manantiales, ríos y cuevas; la presencia de ofrendas y sus motivaciones; el simbolismo de colores; la conexión con conceptos de ancestralidad, y los nexos estrechos con género, a menudo masculino, y estatus. Lo que efectivamente se entiende por «sagrado», sin embargo, depende de una alta especificidad cultural, que puede variar aun en grupos que comparten la misma cultura. Además de ello, cambia con el tiempo, tanto en conceptos como en el uso ritual, lo que le atribuye un carácter dinámico. (cf. Carmichaelet al. 1994 en Kaulicke 2003: 31)

En este trabajo, se analiza dos lugares específicos que tuvieron un proceso de desarrollo cultural primero relacionado a la cultura Cańari y luego al dominio Inca expresado en la presencia de una arquitectura en roca. La entrada para esta investigación en cuanto al estudio de estas determinadas estructuras rocosas, no se limitará a patrones de asentamiento sino a un complejo entramado cultural que da cuenta de una 
cosmovisión del paisaje ligada a estrategias de dominio político e ideológico sobre culturas locales.

Por lo tanto, se ha establecido una metodología que consiste en la identificación de espacios sagrados a través de algunas características planteadas por Criado (1998) y complementadas a través de postulados específicos para las culturas andinas desde la etnohistoria, etnografía y toponimia.

PROPUESTA PARA IDENTIFICACIÓN DE ESPACIOS SIMBÓLICOS EN SOCIEDADES ANDINAS

Postulados Criado (1998):

- Este punto coincide con un accidente geográfico significativo, generalmente una montaña o cumbre prominente y de morfología peculiar, lo que permite su individualización en el conjunto del relieve del que forma parte y desde considerable distancia.

- Existencia de elementos arqueológicos de tipo ritual.

- El carácter especial del sitio se puede reflejar en la toponimia; es incluso posible que ésta recoja términos antiguos significativos.

- Puede existir una tradición popular o folklore en el que se refleja el carácter de la zona o, incluso, se justifica y legitima éste a nivel mitológico.

- Presencia de una santuario o ermita con una tradición especial de prestigio supralocal, generalmente unido a la celebración de festividades importantes, que implican desplazamiento de gente.

Postulados complementarios:

- Cercanía a fuentes de agua: culto al agua

- Relación con otras huacas

Un sitio puede ser sagrado por diversos motivos. Pero para esta investigación, y como segundo eje dentro de esta metodología, se propone que estas estructuras jugaron un rol muy importante como articuladoras de un cambio cultural, para nuestro caso, producto de una conquista.
Las estrategias de dominio de una cultura sobre otra presentan rasgos que se hacen visibles en el paisaje natural. Hay que recordar que la presencia de elementos naturales, ya sea montańas, afloramientos rocosos, lagunas, etc., es un fenómeno natural. Pero la elección de uno de estos como parte de un elemento simbólico es un hecho cultural (Martínez 1998: 546).

Para esto, se propone algunos parámetros que los sitios de este estudio deben cumplir para poder hablar de una intencionalidad referente a estrategias políticas e ideológicas de dominio, como son:

\section{IDENTIFICACIÓN DE RASGOS DE DOMINIO INCA}

1. Material cultural local: en este caso la cultura Cañari

2. Material cultural sociedad conquistadora: en este caso Inca

3. Visibilidad interna y externa

4. Relación al Sistema Vial Andino (Qhapaq Ñan)

5. Cercanía a centros administrativos

6. Relación con elementos de orden mítico de la sociedad conquistadora

7. Cambio en la toponimia

8. Reutilización de sitios estratégicos

\section{Resultados}

La ubicación geográfica de la región donde se llevó a cabo la investigación, actualmente corresponde a la división política del territorio ecuatoriano: cantón El Tambo y cantón Cañar. La provincia del Cañar se encuentra al Sur del Ecuador en la región geográfica denominada Sierra Sur. Colinda con las provincias de Chimborazo al Norte, Azuay al Sur, Guayas al Oeste y Morona Santiago al Este. La zona de estudio se encuentra dentro de la hoya de Cańar, integrada por varios sistemas montańosos como el nudo del Azuay, la cordillera Central, el nudo de Curiquingue-Buerán y una parte de la cordillera occidental (Siguencia 2007: 


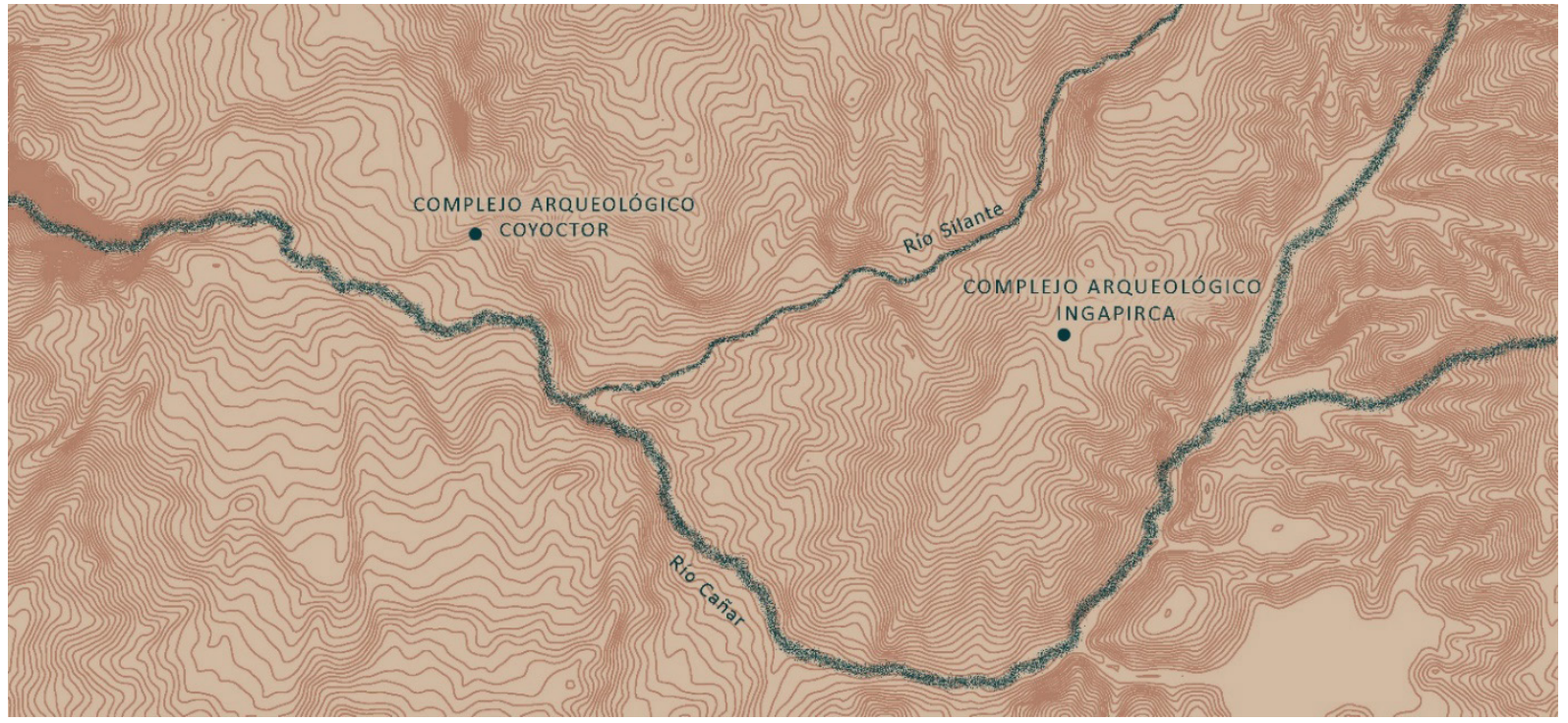

Figura 1: Ubicación de los sitios arqueológicos de Coyoctor e Ingapirca.

14). Precisamente, en la falda sur del nudo del Azuay, en una especie de terraza del valle del río Cañar, al norte de la provincia, se ubican los dos sitios en cuestión. Se trata de una depresión alargada en dirección sureste-noroeste, cuyas laderas están formadas por una serie de terrazas escalonadas dispuestas a ambos lados del cauce del río, siendo las de El Tambo, las más anchas (Sauer 1975 en Siguencia 2007: 15). El área limita al norte con las estribaciones del nudo del Azuay y al Sur con el río Cañar, al oriente por las quebradas Chaupurcu, Puyal y Chantón, y al occidente por el río San Antonio.

\section{Baño del Inca de Ingapirca}

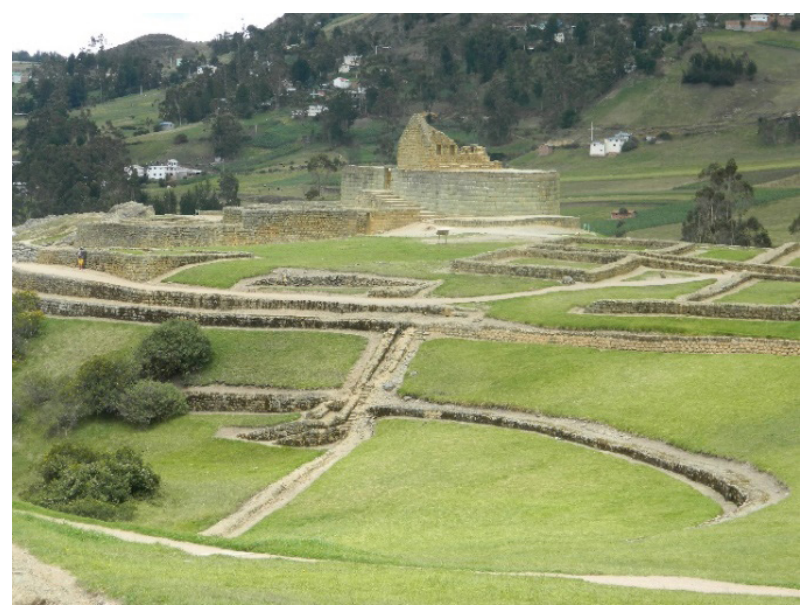

Complejo arqueológico Ingapirca.

\section{Espacios simbólicos}

\section{Primer criterio:}

Entre el río Silante y el río Huayrapungo (cabecera del río Cañar) se levanta el cerro Cubilán en cuya ladera occidental se encuentran las ruinas incaicas de Ingapirca» (Fresco 1984: 38). Así mismo, existen otras lomas cercanas al sitio arqueológico Ingapirca que son Chicho-Chuguín y Silante-Chiripungo (Burgos 1992: 65).

\section{Segundo criterio:}

Los Baños del Inca forman parte del complejo arqueológico de Ingapirca, que es a su vez un conjunto

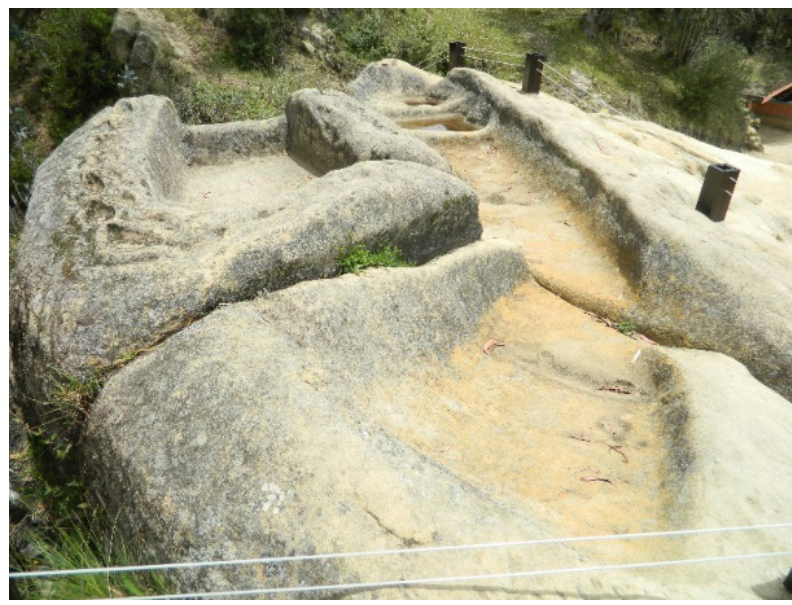

Baño del Inca de Ingapirca. 
de estructuras monumentales de carácter ritual, habitacional y agrícola. La evidencia de la estructura Inca conocida como El Castillo, es la prueba innegable que se trata de un complejo tanto administrativo como ritual. Así mismo, como lo establece Alcina (1978: 144), debió existir una pacarina situada debajo del Castillo y "seguramente también el Ingachungana", acentuando el valor religioso del lugar.

Además, existe una tumba Cañari de una mujer de alto rango junto a 10 esqueletos y con un rico ajuar funerario demuestra que el lugar era sagrado (Alcina 1978). El tipo de enterramiento transmite el estatus de esta persona ya que implica una alta inversión de energía humana en la construcción de la tumba, organización para la preparación de los muertos y colocación de los elementos del ajuar funerario (Binford 1971). La presencia de concha Spondylus estaría ratificando la jerarquía social y por lo tanto el carácter de espacio simbólico.

\section{Tercer criterio:}

Al ser considerados los Incas descendientes del Sol, y ser precisamente el Inca el gobernante del imperio, los sitios que contengan las palabras "Inti» e "Inga» pueden ser considerados lugares con un aspecto simbólico importante debido a su significado tanto político como sagrado. Los Baños del Inca se encuentran en un sitio llamado Ingapirca que quiere decir "Muros del Inca». Así mismo, la quebrada sobre la cual se encuentra esta estructura es conocida como Intihuayco.

Además, existe un pequeño sector ubicado al oeste del Castillo de Ingapirca, siguiendo la quebrada de Intihuayco, frente a los Baños del Inga, conocida como «Intiloma» (Jadán 2010: 18).

\section{Cuarto criterio:}

El Templo del Sol descrito por Cieza de León, que se creía estaba en la supuesta «ciudad de Tomebamba» o sea Pumapungo, no se encuentra allí sino en Hatun Cañar, provincia de Tomebamba (actual Ingapirca). Cieza manifiesta que le informaron los orejones del Cuzco, que eran los más sabios del Imperio, que Tupac Yupanqui fue el fundador del Templo y que se holgaba de estar más tiempo en estos aposentos que en otra parte. (J.L Espinoza 2010: 12).
En esta cita, se puede apreciar la importancia de la estructura conocida como Templo del Sol en Ingapirca para los habitantes de la zona ya que, fue construida directamente para el Inca Túpac Yupanqui (Cieza de León en Espinoza, 2010). El hecho que éste pasase tiempo en este lugar ratifica su carácter sagrado, y por ende acentúa el contexto de sitio estratégico en lo político, pero sobre todo religioso.

\section{Quinto criterio:}

Si bien no existe un santuario como tal, en Ingapirca actualmente se realizan varias fiestas y una en especial conocida como el Intiraymi. Esta fiesta, de origen prehispánico, tiene como objetivo agradecer a la Madre Tierra por los productos obtenidos en las cosechas y se la celebra el 21 de junio en el solsticio de verano. Al lugar asisten las 25 comunidades cańaris que comparten la ceremonia ritual de la "Pambamesa», en donde cada una aporta con diferentes productos. Si bien se trata de una celebración que posiblemente fue insertada contemporáneamente, el hecho de escoger este sitio arqueológico frente a otros que se encuentran en las cercanías como Cojitambo, por ejemplo, puede estar indicando una importancia relevante desde la época prehispánica.

\section{Sexto criterio:}

Como se mencionó anteriormente, el sitio arqueológico se encuentra en una llanura elevada circundada por tres ríos: Silante, Gulán y el que baja del hato de la Virgen. Además, precisamente a la altura del Baño del Inca, se encuentra un ojo de agua que parece alimentar los canales de esta estructura. En la actualidad, parece estar seco. Sin embargo, tanto el sitio en general está relacionado a fuentes de agua y en especial la estructura conocida como Baños del Inca.

\section{Séptimo criterio:}

Ingapirca es un sitio Inca monumental debido a los varios componentes que forman parte del complejo. Sin embargo, cerca de éste, se encuentra un sitio de mucha importancia que los estudiosos lo relacionan directamente con el cerro de Yanacauri, en cuyos pies se encuentra el sitio arqueológico de Coyoctor. En efecto,

Fue el propio González Suárez, quien relacionó el contenido sagrado de los dos monumentos; es 
más, fue él quien sospechó una mayor antigüedad para el complejo de Coyoctor, respecto de las ruinas de Ingapirca que parecen más modernas: [...] Recordemos que el grupo de los tres monumentos incásicos (Ingapirca, Ingachungana e Intihuayco) está a no mucha distancia de un sitio sagrado para los Cañaris: ese sitio es el que ahora se llama GuanaCauri: ese sitio debió ser un lugar de culto religioso para los aborígenes de la comarca. (González Suárez, 1968: 1973 en Siguencia 2007: 92)

Además, Ingapirca está relacionado con un cementerio localizado en Pilzhún, un montículo cerca de El Tambo (Siguencia 20017: 93).

\section{Estrategia de dominio Inca}

\section{Presencia de material Cañari e Inca}

En el complejo arqueológico Ingapirca, se ha encontrado durante las excavaciones la presencia de material cultural de filiación Cańari e Inca, respectivamente.

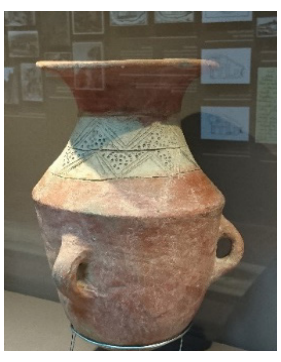

Material cultural Cañari de Ingapirca

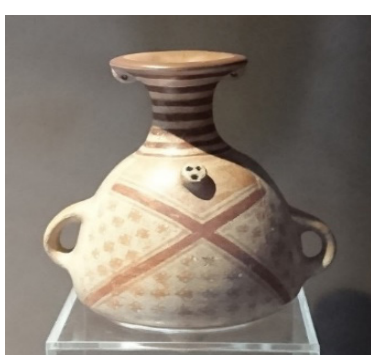

Material cultural Inca de Ingapirca

\section{Visibilidad interna y externa}

La visibilidad es un punto estratégico en cuanto al mensaje de determinada construcción arquitectónica en el espacio. Resulta un factor determinante como lenguaje de dominio. Es por esta razón que se analizó tanto la visualización del sitio desde otros puntos geográficos, así como la visibilidad desde el sitio. Es decir, el campo de visión de elementos naturales que se aprecian desde los Bańos del Inca de Ingapirca permitirá definir el paisaje cultural simbólico.

\section{Relación al Sistema Vial Andino (Qhapaq Nan)}

The Inka road passes the site Ingapirca through cultivated fields on the site's far Eastern side. It is nearly $1000 \mathrm{~m}$ to the west of the famous elliptical structure of fine masonry. Its width is 5-6 m. Surface poterry was common on both sides of the road and there can be little doubt that the raod passed through the site instead of outside it. From Ingapirca the road leads directly south $4 \mathrm{~km}$ to the Cañar (San Pedro) River. (Hyslop 1984: 25)

El Qhapaq Ñan pasa por el sitio arqueológico Ingapirca, lo que demuestra la importancia política y económica de este sitio.

\section{Cercanía a centros administrativos}

Como ya se mencionó anteriormente, los Baños del Inca se encuentran dentro del complejo arqueológico de Ingapirca, en donde la principal estructura, además de monumental, es la elipse conocida como «El Castillo». Este complejo, en su totalidad, corresponde a un centro político y por ende administrativo desde donde el Inca controlaba la región y los avances militares hacia el Norte.

cuando llegaron los Incas, encontraron en Ingapirca un centro de relativa importancia, aspecto este que sumado a ciertas características muy llamativas para ellos, hicieron escoger ese lugar como un centro religioso y administrativo de importancia. (Jaramillo 1976: 22)

\section{Relación con elementos de orden mítico de la sociedad conquistadora}

Cuando Tupac Yupanqui se dirigía a la conquista de Quito, sus hombres descubrieron —según la tradición - en la quebrada llamada Intihuayco una roca que presentaba en una de sus caras lo que ellos creían ser la imagen del sol. Esta imagen, que existe hasta la actualidad, si bien es una formación natural coincidencial como se demostrará en forma amplia, debió construir un llamado a la veneración que por el sol sentía el pueblo del Sur. (González Suárez en Jaramillo 1976: 22)

A través de esta cita, se puede apreciar que Ingapirca está relacionado con elementos de orden mítico para los Incas. Y especialmente, los Baños del Inca, que se encuentran en la parte superior del Intihuayco. 


\section{Cambio en la toponimia}

Cuando llegan los Incas a territorio Cañari, se topan con un asentamiento que era uno de los más importantes para esta cultura. Se trata de "Hatun Cañar» que luego pasa a ser conocido como «Ingapirca», haciendo énfasis en el hecho que se trata de un sitio Inca. Como bien lo recalcan Verneau y Rivet,

Las ruinas que llevan en la actualidad el nombre de Inga-Pirca (Paredes del Inca), se llamaban, en los primeros tiempos de la ocupación española, Hatun-Cañar (El Gran Cañar); [...] Se ubican a 3163 metros de altitud, a $1 \mathrm{~km}$ aproximadamente del encuentro de los ríos Ingapirca y Hato de la Virgen, el cual, 400 metros más abajo, recibe las aguas del Gulan y toma el nombre de Silante» (Traducción mía) (Verneau y Rivet 1912:82).

\section{Reutilización de sitios estratégicos}

Este sitio se ubica en la parte más alta del valle del Cañar por lo que su posicionamiento lo hace particular (Alcina 1978: 144). En este punto es importante mencionar que Ingapirca, en general, está situado en un lugar estratégico geográficamente y puede controlar los intercambios con otras regiones. Al estar en la parte más alta del Valle y en un paso de montaña, puede fácilmente controlar el acceso y la comunicación entre la Costa y la Amazonía (Ibid, 144). El hecho de que los Incas hayan decidido edificar en este lugar un asentamiento político-religioso, reutilizando el mismo espacio Cañari, denota una dominación estratégica.

Además, cerca del sector del Hato de la Virgen, ubicado a $500 \mathrm{~m}$. al Norte del Baño del Inca, existe un camino que habría sido importante desde la época Cañari.

Los rasgos que caracterizan al sitio Hato de la Virgen hacen relación directa con su utilización como cantera en época prehispánica, se encuentra muy cerca a la meseta Guayavilla, Tiene baja incidencia de fragmentos cerámicos, en sus estratos inferiores. Otro elemento que aún se mantiene en este sector es la huella de un camino prehispánico, que al parecer se trata de otro de los ramales del Qhapaq-Ñan que cruza por este sector, ya que aún podemos observar algunos elementos constitutivos de su calzada, así como también de sus pircas late- rales, camino que pone en contacto zonas como Silante bajo, Cajóntambo, Guallavilla, Hato de la Virgen y Sisid. La zona está rodeada además de un sistema de terrazas, andenes los cuales probablemente servían de sustento agrícola, tendiente a mantener el status del centro administrativo ceremonial de Ingapirca. (Jadán 2010: 22)

\section{Baños del Inca de Coyoctor}

Coyoctor es un sitio más complejo ya que presenta una serie de estructuras dispuestas a lo largo del afloramiento rocoso correspondientes al período de Integración y de filiación cultura Inca (1450 y 1532) que sin duda hacen parte de un todo. Es en este contexto, que se puede delimitar el sitio estudiado por la evidencia física encontrada pero también tomando en cuenta al cerro Yanacauri, el cual se encuentra directamente atrás de Coyoctor, siendo parte del paisaje cultural.

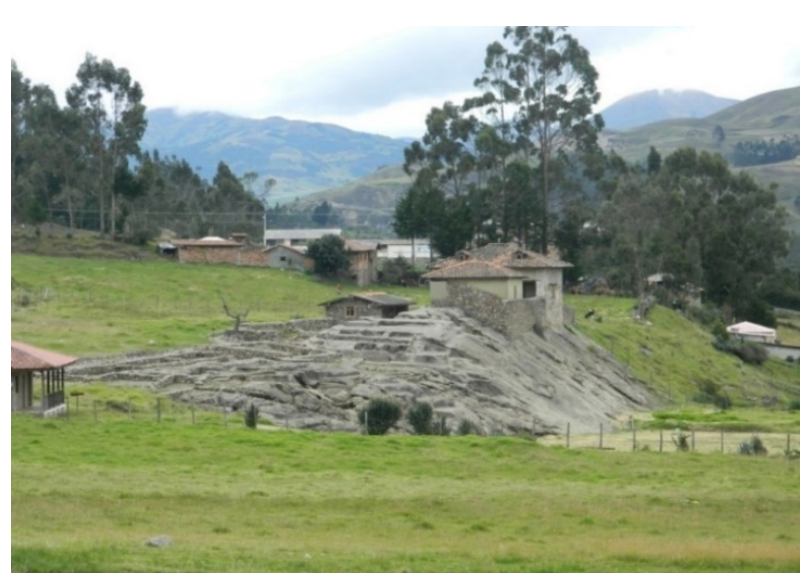

Complejo arqueológico Coyoctor.



Baño del Inca de Coyoctor. 


\section{Espacios simbólicos}

\section{Primer criterio:}

El sitio de Coyoctor se encuentra en las faldas del cerro Yanacauri, además se trata de un afloramiento rocoso que se distingue de forma clara en el paisaje debido a sus dimensiones, y a la explanada que se encuentra en la parte baja.

\section{Segundo criterio:}

Se ha encontrado Spondylus en Coyoctor, como se mencionó anteriormente. Además, es necesario recalcar que «junto al aposento [...] se encuentra un gran monolito que, al parecer, tuvo un carácter funerario, porque cerca de él, los huaqueros lograron desenterrar los restos de un personaje perfectamente engalanado» (Siguencia 2007: 106).

La presencia tanto de Spondylus, que es un elemento ritual, así como de un posible entierro en el lugar, denotan la importancia de Coyoctor en la cosmovisión local.

\section{Tercer criterio:}

La toponimia también provee interesantes datos sobre la zona como los nombres de los cerros que se encuentran encerrando este afloramiento rocoso. Entre ellos, el Yanacauri en cuyas bases se encuentra Coyoctor. Como es de conocimiento común, Yanacauri corresponde al cerro del mito de origen de los Incas.

Como bien lo establecen Verneau y Rivet (1912: 81), para González Suárez «Huana-Cauri es una corrupción de Hának-Huari, palabra a través de la cual se designa los lugares sagrados, en donde, siguiendo la leyenda, nacieron los ancestros de la tribu».

\section{Cuarto criterio:}

El complejo arqueológico Coyoctor está ubicado al pie del cerro Yanacauri, topónimo vinculado al lugar donde los 4 hermanos fundan Cuzco: es decir hay relación con el mito de origen. En este caso, el punto anterior se relaciona directamente con éste ya que la toponimia y las leyendas coinciden.

\section{Quinto criterio:}

Como se vio anteriormente, la etnohistoria no contiene información acerca de este lugar. Mientras que la etnografía arroja resultados positivos. En efecto, en el museo arqueológico de El Tambo, la guía comentó que hasta hace algunos años a finales de septiembre se celebraba la fiesta del Coyaraymi.

En algunas oportunidades, como en la fiesta de la Citua, dedicada al culto de la diosa luna o fiesta de la coya, grupos numerosos de personas que participaban en las festividades, salían del Cusco en peregrinación a las fuentes representativas de cada una de las cuatro provincias del imperio y allí se bañaban, y cambiaban de ropas e insignias para eliminar las enfermedades. (Carrión 2005: 25)

Esta ceremonia, por lo tanto, tenía que ver con el culto a la luna y por ende al agua, en donde se utilizaban fuentes para purificarse.

\section{Sexto criterio:}

Por el sitio pasan dos ríos que se unen en la parte baja y forman el río Cañar. Estos ríos permiten que llegue agua a estos lugares y así se pueda celebrar el culto al agua, evidenciado a través de una serie de canales a lo largo del afloramiento rocoso.

\section{Séptimo punto:}

Coyoctor está en estrecha relación con Ingapirca, ya que se encuentra a menos de $5 \mathrm{~km}$ en línea recta, además de que posee ciertos rasgos parecidos como la talla en afloramiento rocoso, la presencia de piedras sillares, etc.

\section{Estrategias de dominio}

\section{Material cultural Cañari e Inca}

En el Museo Arqueológico del Tambo, existe material cultural de estas dos sociedades que nos indican la presencia Cańari e Inca en el lugar, respectivamente.

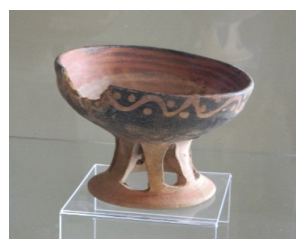

Material cultural Cañari, Museo El Tambo

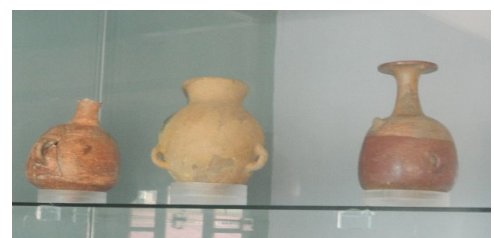

Material cultura Inca, Museo El Tambo

\section{Visibilidad interna y externa}

El sitio se caracteriza por tener una amplia visibilidad desde la parte superior, es decir desde la ladera del 
Yanacauri así como desde la parte inferior, es decir desde la parte plana.

Por un lado, el sitio arqueológico puede ser visto desde todos los ángulos, por lo que no se encuentra escondido, sino que, al contrario, permite ser visto en su totalidad, dándole un carácter especial en cuanto a su importancia de dominio visual. Por otra parte, desde la parte interior de la estructura, la visibilidad es de 360 grados, permitiendo un control completo de los eventos que suceden a su alrededor, como se aprecia en las siguientes fotografías.

\section{Relación al Sistema Vial Andino (Qhapaq Nan)}

Un ramal del Sistema Vial Andino- Qhapaq Nan pasa por el sitio arqueológico, ratificando la importancia a nivel político e ideológico de la estructura.

\section{Cercanía a centros administrativos}

El sitio se encuentra a una distancia de $5 \mathrm{Km}$ de Ingapirca, considerado un centro administrativo y religioso de gran importancia de la cultura Inca.

\section{Relación con elementos de orden mítico de la sociedad conquistadora}

Como se comentó anteriormente, Coyoctor se encuentra al pie del cerro Yanacauri, nombre con el que se identifica al cerro Huanacauri que es en donde se funda Cuzco, de acuerdo al mito de origen Inca. Por lo que esta estructura está en directa relación con la cosmovisión Inca.

\section{Cambio en la toponimia}

Imponían topónimos de su idioma borrando o reemplazando los autóctonos, así lo hicieron con Guapondélic que lo cambiaron a Tumipamba o Coyoctor sustituyéndolo por Huanacauri o Baños del Inca. (Siguencia 2007: 43)

En esta cita, se puede apreciar cambios en los nombres de los lugares a partir de la presencia de los Incas. Además, «[...] los topónimos aplicados a los accidentes geográficos cercanos como Maguana pamba, Yanacauri, Yuto Loma, Cruz Yacu, entre otras evidencias, delatan la evidencia consolidada de la civilización cuzqueña» (Siguencia 2007: 7).

\section{Reutilización de sitios estratégicos}

Otro de los rasgos arqueológicos es el propio camino de acceso que presenta claros rasgos de camino prehispánico probablemente de origen Cañarí, el cual tiene un ancho de aproximadamente $10 \mathrm{~m}$, sus muros laterales no sobrepasan los $0.80 \mathrm{~m}$ de alto siendo estos muros gruesos de cerca de $1 \mathrm{~m}$ de ancho, camino que al parecer comunica las zonas de Yanacauri con Yutuloma y este probablemente con Narrio, ubicado un poco más abajo, tal vez este sea uno de los tantos ramales que parten del QhapaqNan localizado en la parte alta que comunicaban hacia este sector, de igual forma se puede apreciar a un costado de éste unas pequeñas terrazas de sostén de carácter escalonado de forma semi circular, con muros de piedra (canto rodado y arenisca), las cuales al parecer ya tienen una intervención moderna. (Jadán 2010: 42/43)

La descripción de las evidencias arqueológicas alrededor de este sitio demuestra la posición estratégica del lugar. La existencia de un camino Cañari que comunicaba con otras huacas preincas así como el sistema de terrazas, demuestra que la zona era importante. A la llegada de los Incas, éstos se apropian del lugar y reutilizan las vías de acceso y el sistema agrícola implantado.

\section{Discusión}

$\mathrm{Al}$ principio de este trabajo se plantearon algunas preguntas que fueron analizadas a través de una metodología que tomó como base teórica la arqueología del paisaje. A partir de varios criterios expuestos y demostrados para los dos sitios arqueológicos y en base a la información recopilada, se evidencia la importancia de Coyoctor e Ingapirca como paisaje simbólico dentro de una dinámica de dominio político Incaico.

Por una parte, autores como Fresco (1984) y González Suárez (1890) resaltan la cercanía de estos sitios arqueológicos con lugares sagrados por los Cañaris antes de la conquista Inca. El hecho de que los Incas hayan construido este tipo de arquitectura monumental en lugares planificados, nos permite hablar de un palimpsesto cultural, es decir, la im- 
plantación de una nueva forma de ver el paisaje y una reestructuración social y política de la zona. Sin embargo, mediante el análisis toponímico, se puede ver una dinámica que combina los nombres cañaris con la lengua quechua de los Incas permitiendo así entender de qué manera éstos últimos impusieron su dominio ideológico. En Coyoctor, persisten palabras cañaris como "Villagshi», "Shushua», «Chantun» $y$ «Ushcurrún» que designan nombres de fuentes, quebradas y lomas que se encuentran a los alrededores de Coyoctor (esta palabra mismo podría ser de origen local). Sin embargo, dentro de la zona en cuestión, se puede evidenciar nombres quechuas como "Huanacauri», "Cruz Yacu» (combinación de quechua y español) que demuestran la presencia de la cosmovisión Inca. Para el lado de Ingapirca, los nombres Cañaris son pocos como «Gulán», mientras que los nombres quechuas son más abundantes: «Intihuayco», «Ingapirca», «Huayrapungo», «Intiloma» (combinación de español y quechua) y «Chiripungo». Este análisis nos permite interpretar que el dominio Inca en estas dos zonas se vio reforzado por las nuevas designaciones en quechua de los paisajes y zonas geográficas, sobre todo haciendo referencia al sol (Inti), al Inca (Inga), al agua (Yaku) pero sobre todo introduciendo el nombre de Huanacauri que es el lugar en donde se construyó el Cuzco.

Una vez analizado el entorno geográfico, es importante centrar el análisis en el aspecto arquitectónico de estas fuentes Incas. En primer lugar, su posición geográfica es estratégica ya que están en sitios relativamente aislados desde donde la visibilidad tanto de afuera como de adentro es amplia, lo que transmite un sentido de exclusividad y respeto. $\mathrm{Al}$ ser sitios situados en lugares apartados puede suponerse que se llevaban actividades de carácter ritual relacionados al agua debido a la presencia de canales, fuentes y asientos. Este dominio Inca por lo tanto no sólo se evidencia a través de la toponimia pero también en el cambio físico del paisaje natural, en donde a través del trabajo en piedra, en el que se observa la dualidad y la cuatripartición típicas de la cosmovisión Inca, se pone de manifiesto el nuevo significado de este espacio geográfico.

«Periódicas romerías se hacían a las lagunas y manantiales, donde se realizaban importantes ceremonias destinadas a conseguir agua y buenas cosechas. A estas llegaba la "pareja» de niños o adolescentes que personificaban al sol y la luna. En las leyendas del Sur andino, $[. .$.$] , la pareja va a romería a varias fuentes$ (la de Huanacaure, Calispuquio y Yavirá): él portando símbolos de poder y fuerza como la honda y la alabarda, y ella, el cántaro de chicha o de agua, símbolo de las lluvias y de la fertilidad» (Carrión 2005: 23).

En esta cita, recalca el nombre Huanacauri, en donde, según la autora, se realizaban ceremonias al agua. Este hecho es importante ya que ratifica a Coyoctor como un sitio sagrado, con todas las características de un lugar en donde los Incas realizaban cultos propios de su cosmovisión. Pero también, la pareja de niños empleado en esta ritual, puede vincularse con el mito de origen Inca, reforzado por la celebración de la Coyaraymi. Esta fiesta era aún celebrada hace unos cuantos años en Coyoctor.

$\mathrm{Al}$ respecto, es importante detenerse para analizar esta fiesta más a profundidad, ya que es un componente importante de esta investigación pues apoya las hipótesis planteadas.

En «Los Incas y la fiesta de la Situa» de Luis Monteverde (2011), se detalla la fiesta de la Situa (o Coyaraymi), descrita en el relato de Cristóbal de Molina, de a acuerdo a tres componentes: la organización espacial y social en Cuzco durante la fiesta, los posibles antecedentes del empleo y manejo del espacio, de las estructuras, funciones y cultos religiosos; $y$, el manejo y control político-ideológico por parte de los Incas (Ibid: 243).

La fiesta de la Situa se festejaba en agosto o septiembre, siempre después del mes de la siembra y al inicio de la época de las lluvias en tres templos principales de Cuzco (Coricancha, Quishuarcancha y Pucamarca), es decir en espacios abiertos. Sin embargo, tanto Molina (ca. 1574 en Monteverde 2011) como Garcilaso (1609 en Monteverde 2011) señalan al cerro Huanacaure, como un sitio importante durante la Situa y al cual se llevaban ofrendas (Ibid: 243). Esta fiesta buscaba purificar los males producidos con el inicio de las lluvias, por lo que el autor senala varias actividades que se realizaban durante este período, de las cuales resaltan el lavarse para sacarse los males y enfermedades y la ofrenda de sanco (mazamorra de maíz) a la huaca de Huanacaure (Ibid: 244). Bauer y Reid (2015: 218) resaltan también la importancia de la sangre de los camélidos que eran sacrificados durante el rito. Es importante recalcar que esta ceremonia no fue únicamente de tinte sim- 
bólico de purificación, que era una parte importante del ritual, sino que también enfatizó en las alianzas político-religiosas (Ibid: 250).

La religión, justificadora del poder del Inca y de su gobierno, no fue usada como represión social, sino más bien fue el soporte vertebral y justificatoria de las acciones políticas, administrativas, etc., del Tahuantinsuyo. (Monteverde, 2011: 251)

Esta idea es apoyada por Bauer y Reid (2015: 209), quienes caracterizan al ritual de la Situa como un evento de purificación, pero también construida mediante la metáfora de la guerra para justificar las acciones del Estado.

Pero quizá el aspecto más importante de este texto de Monterverde (2011) es el hecho de que:

Algo que comparten el Sol, Viracocha Pachayachachik y Huanacaure, en las fuentes escritas coloniales, es que todos ellos están relacionados con los mitos de origen de los Incas. Mitos que serían celebrados durante la Situa. (Ibid: 251)

Por lo tanto, todo este análisis nos lleva a plantear que los Bańos Incas de Coyoctor e Ingapirca, como se los conoce y que son parte de estos dos complejos arquitectónicos, son la evidencia de un proceso de dominio tanto político como ideológico sobre la cultura Cañari, llevado a cabo a través de cambios simbólicos y físicos del paisaje natural.

\section{Conclusiones}

El trabajo de canales, pozos y fuentes en afloramientos rocosos en zonas importantes para la cultura local, y la evidencia de rasgos ideológicos Incas, permiten establecer claramente que se trata de zonas de culto al agua y por ende de purificación desde donde el Inca legitimaba su dominio a través del mito de origen y la implantación de fiestas religiosas. Es posible que estas fuentes Incas de Ingapirca y Coyoctor fueran construidas por Tupac Yupanqui en su afán de dominar el área conquistada durante sus primeras incursiones en el actual territorio ecuatoriano. Estos puestos de avance hacia el norte de Tomebamba (actual Cuenca) y por ende hacia territorio Puruhá se fueron convir- tiendo en lugares importantes en el aspecto religioso, lugares desde el cual el Inca ratificaba su dominio mediante la recreación de los elementos propios del mito de origen. Si bien Tomebamba constituyó el centro político de los Incas, Ingapirca y Coyoctor, por lo tanto, fueron centros religiosos asentados en antiguas huacas cańaris.

Todos estos aspectos nos llevan a la conclusión que esta región de estudio fue parte de una estrategia de replicar la idea de "Otro Cusco» ${ }^{2}$ en el Chinchaysuyo, truncada por la conquista española, pero que aún conserva los espacios físicos de dominio Inca. Este "Otro Cusco» presenta los mismos rituales ligados a estructuras de poder y legitimación del Estado Inca sobre las sociedades conquistadas, además de la presencia de un cerro denominado "Huanacauri» en el cual se daba, por un lado, la conexión entre el Inca y los dioses andinos, y por el otro, el manejo y control visual del espacio (Apaza y Bolívar 2016: 166/192). Es importante recalcar que el tipo de arquitectura del monumento conocido como Castillo en el Complejo Arqueológico Ingapirca tiene similitud con la curvatura de los muros del Qoricancha en Cusco.

El ritual de la Situa constituye uno de los más importantes en este proceso vinculado a la purificación y la idea de guerra como punto central de las acciones rituales que permitían posicionar a los Incas en la cúspide del mundo, únicamente bajo el sol. La fiesta de la Situa, por lo tanto, «es un modelo para entender cómo la ideología, el poder y el ritual están unidos y son un reflejo de los cambios culturales» (Bauer y Reid 2015: 223. Traducción mía). Las fuentes Incas de Coyoctor e Ingapirca son lugares que evidencian este proceso de dominio Inca tanto en el aspecto político como ideológico y que se debieron a un único propósito: legitimar la conquista e implantar la recreación del mito de origen mediante construcciones en afloramientos rocosos y transformando el paisaje natural de acuerda a su cosmovisión.

2 «...debían establecer nuevamente un centro para generase un nuevo sistema, enmarcándose en la complejidad del sistema estructural del estado Inka en todo su territorio; para establecer el punto nuclear, éste debía poseer las características conceptuales o equivalentes al Cusco, edificando y planificando otros Cusco, que garantizara el control y manejo del mundo andino Inka» (Apaza y Bolívar 2016: 167) 


\section{Bibliografía}

Alcina, Franch (1978). Ingapirca: arquitectura y áreas de asentamiento. Revista Espańola de Antropología Americana. Universidad Complutense de Madrid. España.

Alonso GonzÁlez, Pablo (2009). «Arqueología contemporánea y de la postmodernidad: Gestión e Interpretación en val de San Lorenzo (León)». Arqueoweb. Revista sobre arqueología en internet. Universidad de León.

Apaza, John; Bolívar, Wilber (2016). «El Otro cusco: Choquequirao» en Revista Arqueología y Sociedad N. 31, pp. 165-196. UNMSM. Lima.

Bauer, Brian; Reid, David (2015). "The Situa ritual of the Inca. Metaphor and performance of the State» en Perspectives on the Inca, Monica Barnes, Inés de Castro, Javier Flores Espinoza, Doris Kurella, and Karoline Noack (eds.), pp. 208-225. Stuttgart: LindenMuseum, Sonderband/Tribus.

Binford, Lewis (1971). «Mortuary Practices: Their Study and Their Potential» en J.A. Brown (ed.) Approaches to the Social Dimensions of Mortuary Practices. Memoirs of the Society for American Archaeology 25, Pp 6-29.

Burgos, Hugo (1992). "El mito del urcuyaya» en El mito en los pueblos indios de América: actualidad y pervivencia. Colección 500 años. No. 48. Cayambe, Ecuador.

CARrión CACHOt, Rebeca (2005). El culto al agua en el antiguo Perú. Segunda Edición, Instituto Nacional de Cultura. Perú.

Constantine, Angelo (2013). Rumipamba bajo la sombra del Pichincha. Ampliación del Estudio de complementación de datos actualísticos Parque Arqueológico y Ecológico Rumipamba. Informe final entregado al Instituto Nacional de Patrimonio Cultural. Quito.

Criado, Felipe (1998). Arqueología del Paisaje. Seminario de Arqueología y Etnología Turolense. Instituto de Estudios Turolenses, España.

Criado, Felipe (1999). «Del terreno al espacio: planteamientos y perspectivas para la arqueología del paisaje» en CAPA, 6. Universidad de Santiago de Compostela. España.

Espinoza, José Luis (2010). «Tomebamba, Pumapungo, Hatun Cañar» en Apachita No. 16, Boletín del área de Arqueología. PUCE. Quito.

FresCo, Antonio (1984). La arqueología de Ingapirca: costumbres funerarias, cerámica y otros materiales. Comisión del castillo de Ingapirca. Quito.
González SuÁrez, Federico (1890). Historia General de la República del Ecuador. Tomo I. Publicaciones Educativas Ariel. Quito.

Granja, Abigail (2014). Los petroglifos del Catazho, Morona Santiago una aproximación a su estudio desde la arqueología del paisaje. Tesis para obtención del Grado en Antropología con mención en Arqueología. PUCE. Quito.

Hyslop, John (1984). The Inka Road System. Institute of Andean Research. Academic Press, Inc. New York.

Hodder, Ian (1990). «Reading the past. Current approaches to interpretation in archaeology». Cambridge University Press, Cambridge.

Jadán, María (2010). Delimitación Participativa de los Paisajes Arqueológicos de Ingapirca y sus alrededores. Informe Final entregado al INPC. Cuenca.

Jaramillo, Mario (1976). Estudio histórico sobre Ingapirca. Pontificia Universidad Católica del Ecuador. Quito

Kaulicke, Peter., et al. (2003). «Agua, ancestros y arqueología del paisaje» en Boletín de arqueología PUCP. N. 7, pp. 27-56. Perú.

MartíneZ, Julián (1998). "Abrigo y accidentes geográficos como categorías de análisis en el paisaje de la pintura rupestre esquemática. El sudeste como marco». Seminario de Arqueología y Etnología Turolense. Instituto de Estudios Turolenses, España.

Monteverde Sotil, Luis (2011). «Los Incas y la fiesta de la Situa» en Chungara, Revista de Antropología Chilena. Volumen 43, No2, Pp. 243-256. Chile.

Preucel, Robert (1990). Between Past and Present: Issues in Contemporary Archaeological Discourse. Carbondale: Southern Illinois University Press.

SAUER, Carl(1925). «TheMorphologyoflandscape». University of California, Publications in Geography 2. USA. Schuster Fonseca, Juan (1993). "Teoría de la Estructuración» Período Jul-Sep: 87. Pp.97-107. México.

Siguencia, Rolando (2007). Coyoctor. Recinto Sagrado. Casa de la Cultura Ecuatoriana, Azogues.

Tilley, Christopher (1990). The art of ambiguity: Material Culture and Text. Routledge. London.

Ugalde, María Fernanda; Constantine, Angelo: Chacón, Rosalba (2009). «Persistencias y rupturas en el uso del espacio. Reflexiones sobre identidad y áreas de actividad» en: Antropología. Cuadernos de investigación (8): 105-119. C. Landázuri (ed.). PUCE, Quito.

Verneau, Rene; RIVET, Paul (1912). Ethnographie ancienne de l'Equateur. Paris. 